\title{
DEM Simulation of Particle Stratification and Segregation in Stockpile Formation
}

\author{
Dizhe Zhang ${ }^{1}$, Zongyan Zhou ${ }^{1 . *}$, and David Pinson ${ }^{2}$ \\ ${ }^{1}$ Laboratory for Simulation and Modelling of Particulate Systems (SIMPAS), Department of Chemical Engineering, Monash \\ University, VIC 3800, Australia \\ ${ }^{2}$ BlueScope Steel, Port Kembla, NSW 2505, Australia
}

\begin{abstract}
Granular stockpiles are commonly observed in nature and industry, and their formation has been extensively investigated experimentally and mathematically in the literature. One of the striking features affecting properties of stockpiles are the internal patterns formed by the stratification and segregation processes. In this work, we conduct a numerical study based on DEM (discrete element method) model to study the influencing factors and triggering mechanisms of these two phenomena. With the use of a previously developed mixing index, the effects of parameters including size ratio, injection height and mass ratio are investigated. We found that it is a void-filling mechanism that differentiates the motions of particles with different sizes. This mechanism drives the large particles to flow over the pile surface and segregate at the pile bottom, while it also pushes small particles to fill the voids between large particles, giving rise to separate layers. Consequently, this difference in motion will result in the observed stratification and segregation phenomena.
\end{abstract}

Keywords: stockpile, segregation, stratification, DEM

\section{Introduction}

The pattern formation of particle stockpiles is primarily a practical concern. During the stockpiling process, segregation and stratification happen spontaneously and directly lead to the de-mixing state of the particulate system. Therefore, in order to increase the stockpiles' mixing quality, it is of great significance to have a better understanding of these two phenomena.

It is already acknowledged that segregation and stratification are both induced by the difference of particle properties among granular mixtures. For segregation, significant investigation of its mechanisms has been undertaken. Williams [1-3], and Drahun and Bridgwater [4] experimentally studied the heap flow segregation using binary mixtures of spherical particles. They demonstrated a percolation mechanism where small particles tend to sink into the voids while large particles are able to float onto the surface and roll down to the pile bottom. For stratification, many researchers have reported this feature with the use of particles in different shapes. They also further proposed several mechanisms to explain this phenomenon. Makse et al. [5-7] believed that stratification is induced by a "kink" mechanism and the key parameter controlling the process is the repose angle of different species. Gray et al. [8-10] experimentally proved that surface avalanches are inducing the stratification. From their work, avalanches work as a kinetic sieve which triggers the particle layering, while dispersed shock waves can further bring the granular matters rapidly to rest. This theory was also extensively studied by Baxter et al. [11] with poured granular heaps. Different from the previous researchers, Benito et al. [12] experimentally demonstrated the existence of stratification using only spherical particles, and they further conducted parameter studies in an attempt to determine the parameter that initiates stratification.

In this article, we present a numerical study of spontaneous segregation and stratification of binary spherical particles when forming a stockpile. With the use of a discrete element model, both of these phenomena were observed and a comprehensive view of the particle movements was obtained. After a detailed parameter study of size ratio, injection height and mass ratio, Benito et al.'s findings $[12,13]$ on stratification were validated and further the effects of these parameters on stockpile mixing quality were demonstrated. Of the mechanisms dominating the pattern formation, we found that stratification is induced by the void-filling mechanism, while further pile avalanches and motion of large particles will result in segregation.

\footnotetext{
* Zongyan Zhou: zongyan.zhou@monash.edu
} 


\section{Simulation method and conditions}

Simulations were conducted based on a DEM model previously developed by Zhou et al.[13]. The simulated problem is shown schematically in Fig. 1. The device includes two parts: a particle injection device and a receiving box $(200 \mathrm{~mm} \times 100 \mathrm{~mm} \times 5 \mathrm{~mm})$. During the growth of the stockpile, the distance between injection surface and top of stockpile (injection height) was set constant by moving the injection surface vertically during the stockpile formation. Specifically for this work, the injection height is set as either $10 \mathrm{~mm}$ or $50 \mathrm{~mm}$ to study its effects on pattern formation.

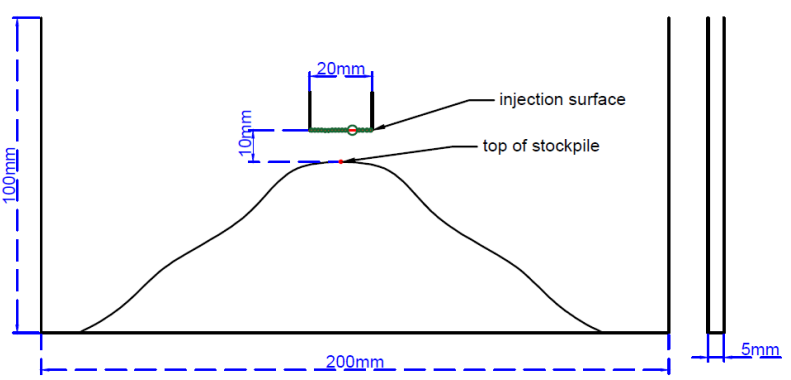

Fig. 1 Schematic diagram of the simulation conditions. (Injection surface: the surface where particles are generated and released; top of stockpile: the highest point of the formed stockpile)

The binary granular mixtures used in the simulations are formed by spherical particles of two different size ratios ( $1 \mathrm{~mm}$ and $3 \mathrm{~mm}, 2 \mathrm{~mm}$ and $3 \mathrm{~mm}$ ). The mass ratio $R$ (the total mass ratio of the two species which also represents the flow rate ratio of large particles versus small particles) was set to be 0.5 or 1 . Other physical properties of the particles were set to those of glass beads. Details of the input variables and their values are shown in Table.1.

Table 1 Input variables and their values

\begin{tabular}{|c|c|}
\hline Input variables & Value \\
\hline Young's module & $1 \times 10^{8} \mathrm{~N} / \mathrm{m}^{2}$ \\
\hline Poisson's ratio & 0.3 \\
\hline Sliding coefficient (P-P) & 0.4 \\
\hline Sliding coefficient (P-W) & 0.4 \\
\hline Rolling coefficient (P-P) & 0.3 \\
\hline Rolling coefficient (P-W) & 0.3 \\
\hline Particle density & $2.7 \times 10^{3} \mathrm{~kg} / \mathrm{m}^{3}$ \\
\hline Particle number & 20,000 \\
\hline Flow rate & $0.1 \mathrm{~g} / \mathrm{s}$ \\
\hline
\end{tabular}

\section{Results and discussions}

Fig. 2 shows some of the patterns obtained from the simulations. After pouring a well-mixed particle stream into the slot, segregation and stratification spontaneously occur within the stockpiles. These patterns vary when changing the parameters including size ratio, injection height and mass ratio. It is clearly observed in Fig. 2 that the presence of segregation is not strictly limited by the testing parameters. For example, while a size ratio of $3: 2$ is already large enough to trigger stratification, stratification is only observed in cases with size ratios exceeding 3:1 combined with a low injection height. In the following sections, parameter effects in terms of the mixing quality and mechanisms of stratification and segregation will be discussed in detail.

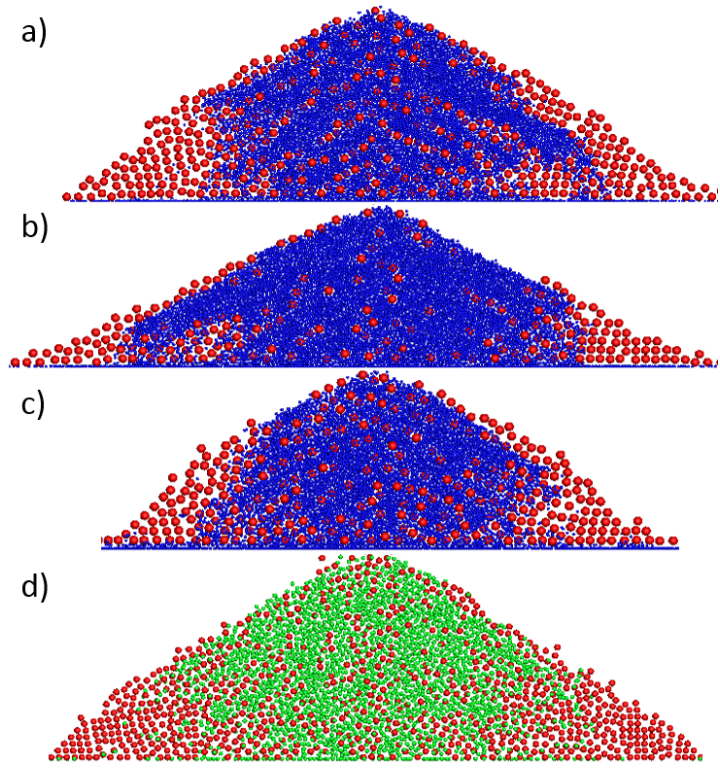

Fig. 2 Stockpile patterns of different cases : a) size ratio $3: 1$, injection height $10 \mathrm{~mm}$, mass ratio $R=1$; b) size ratio $3: 1$, injection height $10 \mathrm{~mm}$, mass ratio $R=0.5$; c) size ratio $3: 1$, injection height $50 \mathrm{~mm}$, mass ratio $R=1$; d) size ratio $3: 2$, injection height $10 \mathrm{~mm}$, mass ratio $R=1$. Particle sizes have been scaled in the figure (scale factor $=0.7$ ).

\subsection{Mixing quality}

A common method to quantify the mixing quality of a granular mixture is to use the mixing index. In this work, the mixing index $M$, first introduced by Lacey [14] and further developed by Feng et al. [15] was adopted. This index is given by

$$
M=\frac{S_{0}^{2}-S^{2}}{S_{0}{ }^{2}-S_{R}^{2}}
$$

where $S^{2}$ is the actual variance, while $S_{0}{ }^{2}$ and $S_{R}{ }^{2}$ are the variances of the completely segregated and completely mixed states, respectively. So a completely segregated mixture will have $M$ as 0 . More details about this equation can be found in Feng et al.'s work [15].

Fig. 3 demonstrates the comparisons of $M$ among different cases. Firstly, the particle size ratio can effectively influence the pattern formation as large size ratio will induce stronger segregation [4]. Then, for the injection height, it will affect the impact velocity, thus influencing the further particle movement. As shown from Fig. 3, a higher injection point with larger impact velocity 
can slightly reduce the measured segregation. Finally, the mixing quality is also largely affected by $R$ that larger $R$ indicates lower $M$. These results can be explained by the void-filling mechanism as discussed in the following sections.

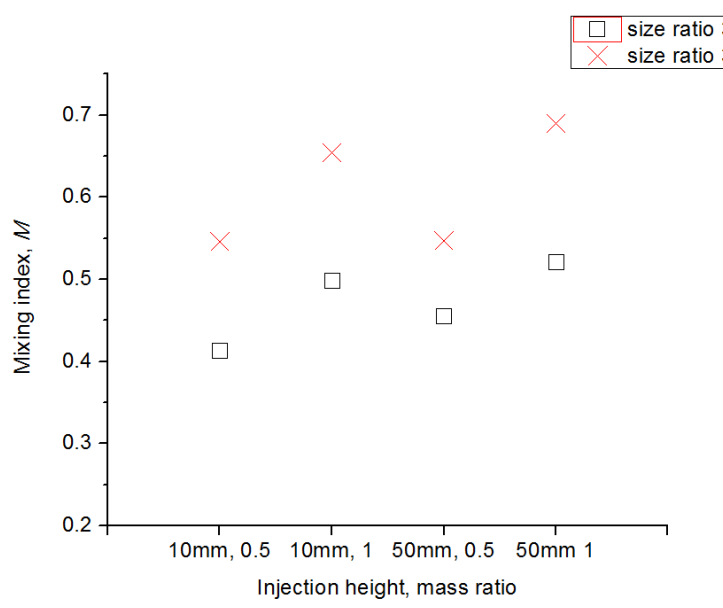

Fig. 3 Effects of size ratio, injection height and mass ratio on mixing quality in terms of mixing index, $M$

\subsection{Stratification}

From Fig. 2, particle stratification is easily observed in Fig. 2a) and Fig. 2b). This layering phenomenon can be explained by a void-filling mechanism which has been used to explain the situations in chute flow and vibrated granular beds $[16,17]$. When particles of different sizes are mixed together within an active system, this mechanism presents the process that gravity and inertia which drives small particles to sink or percolate into the voids between large particles instead of flowing on the particle surface. Fig. 4 shows the specific situation for the stockpiling process. When pouring well mixed granular mixtures onto a flat surface to form a pile, the small particles percolate into the voids while large particles are able to flow on the top of the surface. This difference in motion will then cause the accumulation of small particles between large particles, thus divide the large particles into separate single layers. The lower layer remains motionless while the upper layer is free to slide until the particles reach a "block".

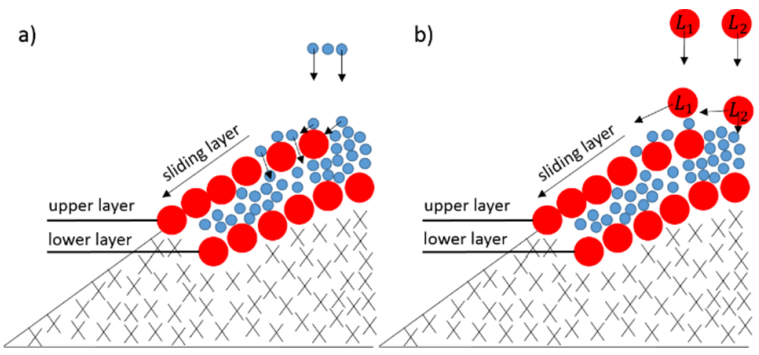

Fig. 4 Comparison of the motion of different particles: a) motion of small particles; b) motion of large particles
The "block" is a special area within the region where the void-filling process occurs. With the growth of the stockpile, available voids for small particles to fill into become fewer and the porosity of the area decreases. When some critical points have extremely low porosity, the void-filling process will then stop. The area with extremely low porosity has a high resistance to flow and represents the "block". Fig. 5 shows the "block" inside the stockpile identified from spatial distribution of porosity. As the "block" stops the small particles' penetration motion, the outer layer of the stockpile becomes more unstable as additional particles drop onto the pile. This instability then causes further avalanche of the outer particles, especially for large particles. This avalanche behaviour consequently reforms the distribution of outer particles and removes the "block", which will then reinitiate the void-filling process and successively regenerate the stratification phenomenon leading to the regular layered stratification pattern.

The regular motion of large particles is shown schematically by particle $L_{1}$ in Fig. 4b). These large particles tend to flow onto the particle surface until reaching the pile bottom or forming the sliding layer until a "block" forms. However, there is still another type of particle motion that should be considered. Sometimes, for large particles like $L_{2}$, their inertia drives them to sink into the pile and subsequent burial by the later particles. Such particles remain motionless within the pile and their presence can be used to explain the irregular distribution of some large particles at the centre area of the stockpile. The mechanisms discussed above can be used to explain the effects of different parameters on stratification. In Fig. 2 , stratification is not observed for cases with large injection height and small size ratio. For large injection height, it will significantly influence the impact velocity when particles drop on the stockpile, as large particles with higher impact velocity will easily percolate into the pile instead of flowing on the particle surface. As mentioned above, this motion will then cause the irregular distribution of large particles within the stockpiles instead of the stratification. For size ratio, as stratification is mainly driven by the void-filling mechanism, available voids can be the most important factor for triggering this process. Lower size ratio will make the voids comparably smaller for small particles which increases the difficulty for them to enter into the available voids. This decrease of available voids will then weaken the void-filling process, thus decrease the stratification.

\subsection{Segregation}

Segregation specifically represents the phenomenon that large particles tend to segregate at the bottom of the stockpile. This phenomenon can be clearly observed from the patterns simulated in Fig. 2.

As shown in Fig. 4, the void-filling mechanism differentiates the motion of particles with different sizes. For large particles, gravity and inertia drive them to flow onto the particle surface, and consequently, large particles will segregate to the bottom of the pile. Similarly, when the particles avalanche following the formation of a 
"block", the outer particles also flow to the bottom. Such avalanches also result in the segregation of large particles as the outer particles are mostly large particles as the small particles are more likely to percolate inside. This finding is also consistent with Drahun and Bridgewater's experiments with free surface flow [4].

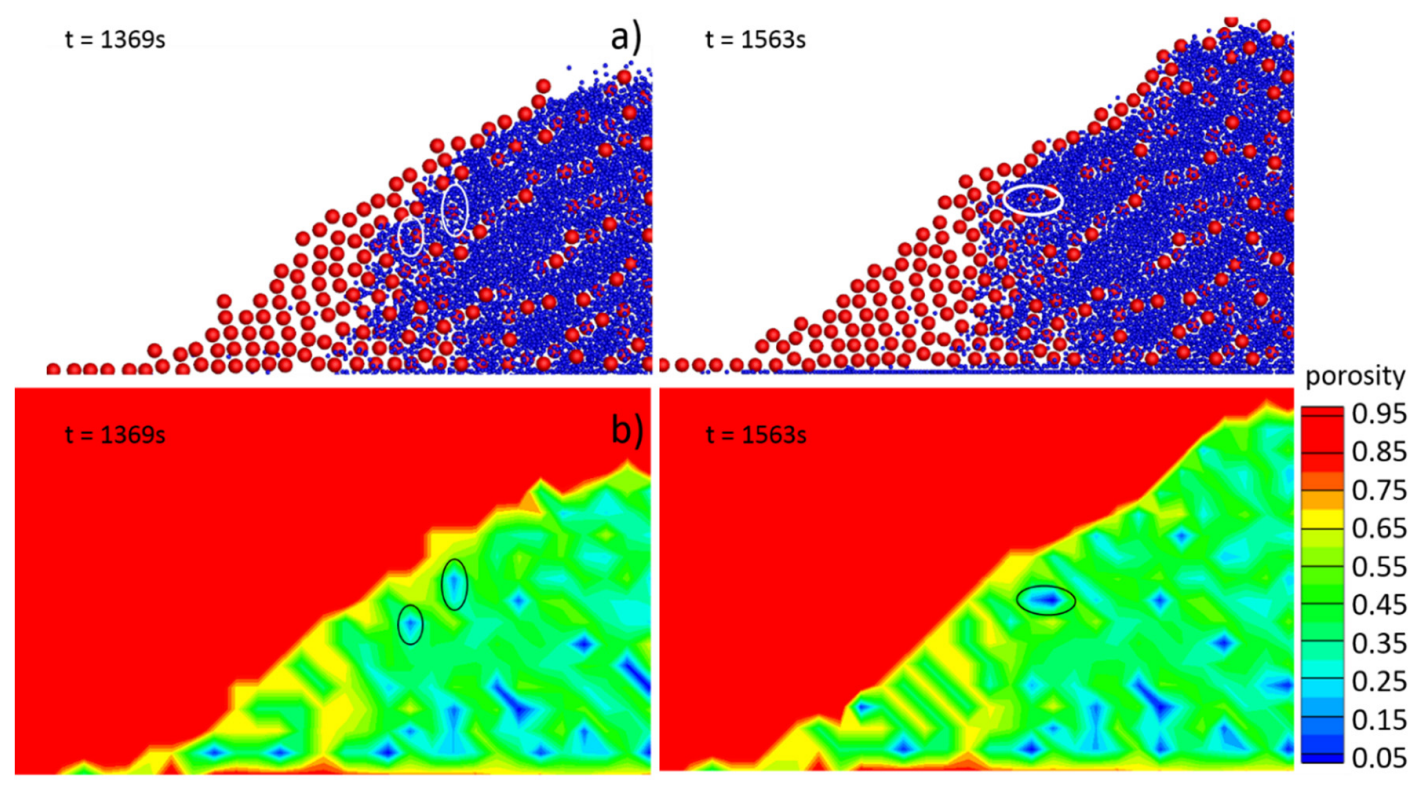

Fig. 5 a) Stockpile particle distribution patterns and b) porosity spatial distribution when the void-filling process stops. The figures are captured at 2 different times $(t=1369 \mathrm{~s}$ and $\mathrm{t}=1563 \mathrm{~s})$; the circled area is where "block" is located; particle sizes have been scaled in the figure (scale factor $=0.7$ ).

\section{Conclusions}

A numerical study based on DEM simulations was carried out to study the stockpile formation in terms of the segregation and stratification processes. The effects of factors including size ratio, injection height, and mass ratio, $R$ were investigated, and a relationship between the mixing quality and these factors was found. A larger size ratio, lower injection height and comparably smaller $R$ can induce stronger segregation, which also represents a lower mixing quality. Furthermore, the stratification phenomenon was successfully simulated with the use of spherical particles, and the simulation conditions and results are consistent with Benito et al.'s physical experiments [12].

In terms of the underlying mechanism causing stratification and segregation, the void-filling mechanism dominates and its effects can explain the occurrence of these two phenomena. This mechanism drives particles of unequal size to move differently, which will then induce the specific phenomena.

\section{Acknowledgements}

This work was financially supported by Australian Research Council (ARC) and BlueScope Steel. Simulations were conducted with the support of NCI National Facility.

\section{References}

1. J. Williams, Fuel Soc. J, 1963. 14: p. 29-34.

2. J. Williams, Powder Technol, 1976. 15(2): p. 245-251.

3. J. Williams, Powder Technol, 1968. 2(1): p. 13-20.

4. J. Drahun, J. Bridgwater, Powder Technol, 1983. 36(1): p. 39-53.

5. P. Cizeau, H. Makse, H. Stanley, Phys Rev E, 1999. 59(4): p. 4408-4421.

6. H. Makse, P. Cizeau, H. Stanley, Phys. Rev. Lett. 1997. 78(17): p. 3298-3301.

7. H. Makse, P. Cizeau, H. Stanley. Physica A, 1998. 249(1-4): p. 391-396.

8. J. Gray, B. Kokelaar, J Fluid Mech, 2010. 652: p. 105137.

9. J. Gray, K. Hutter, Continuum Mech Therm, 1997. 9(6): p. 341-345.

10. J. Gray, C. Ancey, J Fluid Mech, 2009. 629: p. 387423.

11. J. Baxter, U. Tuzun, D. Heyes, I. Heyati, P. Fredlund, Nature, 1998. 391(6663): p. 136-136.

12. J. Benito, I. Ippolito, A. Vidales, Powder Technol, 2013. 234: p. 123-131.

13. Y. Zhou, B. Wright, R. Yang, B. Xu, A. Yu, Physica A, 1999. 269(2-4): p. 536-553.

14. P. Lacey, Chem Eng Res Des, 1997. 75: p. S49-S55. 15. Y. Feng, B. Xu, S. Zhang, A. Yu, P. Zulli, AIChE J, 2004. 50(8): p. 1713-1728.

16. S. Savage, C. Lun, J Fluid Mech, 1988. 189: p. 311335.

17. Arshad, K., Rep Prog Phys, 2004. 67(3): p. 209. 